TITLE:

\title{
Signal estimation and threshold optimization using an array of bithreshold elements
}

$\operatorname{AUTHOR}(\mathrm{S}):$

Sato, AH; Ueda, M; Munakata, T

CITATION:

Sato, AH ...[et al]. Signal estimation and threshold optimization using an array of bithreshold elements. Physical Review E 2004, 70(2): 021106.

ISSUE DATE:

2004-08

URL:

http://hdl.handle.net/2433/50301

RIGHT:

Copyright 2004 American Physical Society 
PHYSICAL REVIEW E 70, 021106 (2004)

\title{
Signal estimation and threshold optimization using an array of bithreshold elements
}

\author{
Aki-Hiro Sato \\ Department of Applied Mathematics and Physics, Graduate School of Informatics, Kyoto University, Kyoto 606-8501, Japan \\ Michihito Ueda \\ Matsushita Electric Industrial Co. Ltd. 3-4, Hikaridai, Soraku-gun, Kyoto 619-0237, Japan \\ Toyonori Munakata \\ Department of Applied Mathematics and Physics, Graduate School of Informatics, Kyoto University, Kyoto 606-8501, Japan
}

(Received 19 February 2004; published 31 August 2004)

\begin{abstract}
We consider the problem of optimizing signal transmission through multichannel noisy devices. We investigate an array of bithreshold noisy devices which is connected in parallel and convergent on a summing center. Utilizing the concept of noise-induced linearization we derive an analytical approximation of the normalized power norm and clarify the relation between the optimum threshold and the standard deviation of noises. We show that the optimum threshold value is 0.63 times the standard deviation of the noises. This relation is applicable to both subthreshold and suprathreshold inputs.
\end{abstract}

DOI: 10.1103/PhysRevE.70.021106

PACS number(s): 05.40.Ca, 02.50.Ey

\section{INTRODUCTION}

Stochastic resonance (SR) has attracted considerable attention of many researchers during the last quarter century [1-7]. At first SR was proposed to explain the observed periodicities in global climate dynamics [1]. SR occurs when the signal-to-noise ratio (SNR) for the response of a single nonlinear system to a subthreshold sinusoidal input signal has its maximum at a nonzero noise strength $D$. As is well known, the SR effect is understood as an enhancement of the system input to a subthreshold input signal by the addition of noise.

There are many studies on SR for a single element. For example, Gammaitoni et al. showed with SNR that a subthreshold sinusoidal signal to a single threshold element is optimally transduced by appropriate additive noise [8]. Collins et al. also reported that a single neuron can optimally transmit a slowly varying subthreshold aperiodic signal with the aid of appropriate additive noise [9]. They proposed the power norm $C_{0}$ and the normalized power norm $C_{1}$ in order to measure a correlation between the input signal and the output signal, and showed that both $C_{0}$ and $C_{1}$ nonmonotonically vary with increasing the noise strength. It is known as aperiodic stochastic resonance (ASR).

Many researchers have both experimentally and numerically studied the symmetrical stochastic resonator, such as the Schmitt trigger [10-13]. In recent years, the central attention of SR seems to move to a network of the stochastic resonators, instead of a single stochastic resonator, such as the global coupled networks and linear chains $[14,15]$. In more recent years a parallel array of nonlinear elements gathers a lot of attention, where the parallel array means that the nonlinear elements are connected in parallel and convergent on a summing center $[6,16,17]$.

Recently, Stocks studied the parallel array of the nonlinear devices and reported that the suprathreshold stochastic resonance (SSR) can be observed on this array motivated by applications to signal processing [6]. Also, from the stand- point of neurophysiology, Collins et al. and Chialvo et al. studied that a parallel array of noisy neurons can exhibit ASR for slowly varying signals $[16,17]$. Consequently, it is important to consider the parallel array in both signal processing and neurophysiology.

The main focus of the article is to find an optimal threshold to transmit an arbitrary signal on the parallel array of the bithreshold elements. According to the assumption that the amplitude of the input signal is smaller than the standard deviation of the noises, we derive an analytical approximation of the normalized power norm. Under this assumption it is not necessary to distinguish between subthreshold and suprathreshold. Furthermore, we apply the linear response theory to the system that we consider in the article. The fundamental idea is the noise-induced linearization, which is an effect that an ensemble average of output from a nonlinear system is linearized due to noise [19]. From the theoretical approximation of the normalized power norm we show that there exists the optimal threshold to maximize it.

In fact, Stocks and Mannella numerically showed that for a summing network of FitzHugh-Nagumo equations, adjusting the threshold to maximize information transmission does not remove SR effects. They pointed out that there is an optimal threshold to maximize the mutual information [18]. Our result is consistent with their indication.

The article is organized as follows. In Sec. II we show the array of bithreshold units. It is well known that Schmitt trigger is a prototype of bithreshold devices [10]. In Sec. III we theoretically derive an approximation of the normalized power norm under the assumption that the norm of the input signal is smaller than the additive noise. Utilizing the approximation we find an optimal threshold where the normalized power norm is maximized. In Sec. IV we perform numerical simulations for the model and show that the threshold value at the maximum normalized power norm depends on the variance of the additive noises. Sec. V is devoted to concluding remarks. 


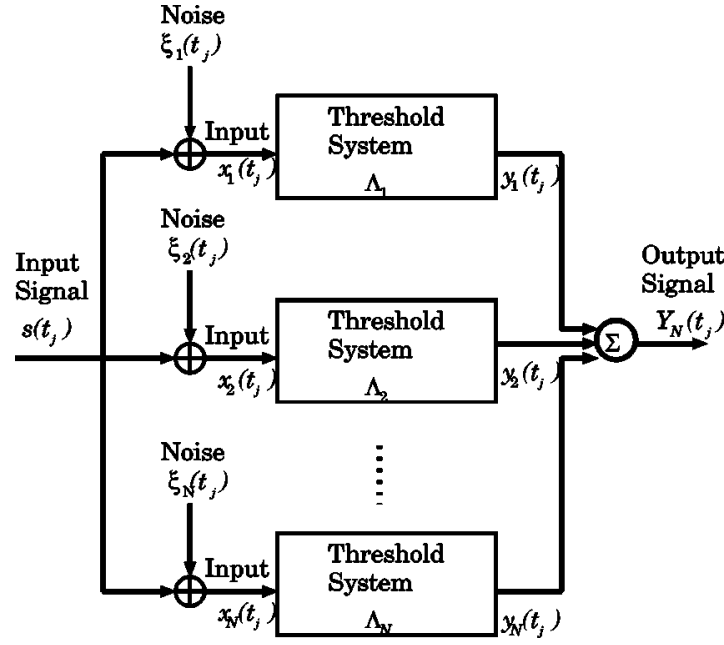

FIG. 1. The array of bithreshold elements with a summing center. $s\left(t_{j}\right)$ exhibits the input of the system. Each subsystem is a bithreshold element, which has three output values. $x_{i}\left(t_{j}\right), y_{i}\left(t_{j}\right)$, and $\Lambda_{i}$ represent the input, the output, and the threshold value in the $i$ th subsystem, respectively. All the output-of subsystems are summed by the summing center and divided by $N . Y_{N}\left(t_{j}\right)$ shows the system output.

\section{MODEL}

Figure 1 displays noisy bithreshold elements connected in parallel and convergent on a summing center. $s\left(t_{j}\right)$, which is sampled with a sampling period $T$, namely, $t_{j}=j T(j$ $=0,1,2, \ldots)$, represents a weak aperiodic signal fluctuating around 0 with $x_{i}\left(t_{j}\right)$ and $y_{i}\left(t_{j}\right)$ denoting the input to and output from the $i$ th subsystem, respectively. The input to the bithreshold element is transmitted over noisy channel. Hence, the input to the subsystem is expressed as

$$
x_{i}\left(t_{j}\right)=s\left(t_{j}\right)+\xi_{i}\left(t_{j}\right),
$$

where $\xi_{i}\left(t_{j}\right)(j=0,1,2, \ldots)$ is independently sampled from the Gaussian distribution,

$$
p_{i}(\xi)=\frac{1}{\sqrt{2 \pi D_{i}}} \exp \left(-\frac{\xi^{2}}{2 D_{i}}\right),
$$

where $D_{i}>0$ are the variance of $\xi_{i}\left(t_{j}\right)$. Each bithreshold element is symmetric and has three output values. It is formalized by

$$
y_{i}\left(t_{j}\right)= \begin{cases}1 & \left(x_{i}\left(t_{j}\right)>\Lambda_{i}\right) \\ 0 & \left(-\Lambda_{i} \leqslant x_{i}\left(t_{j}\right) \leqslant \Lambda_{i}\right) \\ -1 & \left(x_{i}\left(t_{j}\right)<-\Lambda_{i}\right)\end{cases}
$$

where $\Lambda_{i}>0$ are threshold values.

The system output through the summing center $Y_{N}\left(t_{j}\right)$ is defined as

$$
Y_{N}\left(t_{j}\right)=\frac{1}{N} \sum_{i=1}^{N} y_{i}\left(t_{j}\right)
$$

where $N$ is the number of the subsystems. Without noises, each input $x_{i}\left(t_{j}\right)$ cannot cross the threshold value, leading to the system output $Y_{N}\left(t_{j}\right)=0$. With noises having an appropriate variance, $x_{i}\left(t_{j}\right)$ can cross the threshold value.

\section{THEORETICAL ANALYSIS}

We consider the $i$ th bithreshold subsystem. Let $P_{+}\left(t_{j}\right)$, $P_{-}\left(t_{j}\right)$, and $P_{0}\left(t_{j}\right)$ be probabilities that $y_{i}\left(t_{j}\right)$ takes $1,-1$, and 0 , respectively. For an arbitrary input signal $s\left(t_{j}\right)$ these probabilities are given by

$$
\begin{aligned}
& P_{+}\left(t_{j}\right)=\frac{1}{2} \operatorname{erfc}\left(\frac{\Lambda_{i}-s\left(t_{j}\right)}{\sqrt{2 D_{i}}}\right), \\
& P_{-}\left(t_{j}\right)=\frac{1}{2} \operatorname{erfc}\left(\frac{\Lambda_{i}+s\left(t_{j}\right)}{\sqrt{2 D_{i}}}\right), \\
& P_{0}\left(t_{j}\right)=1-P_{+}\left(t_{j}\right)-P_{-}\left(t_{j}\right),
\end{aligned}
$$

where $\operatorname{erfc}(x)$ is the complementary error function, defined as

$$
\operatorname{erfc}(x)=\frac{2}{\sqrt{\pi}} \int_{x}^{\infty} e^{-u^{2}} d u
$$

For simplicity we set $\Lambda_{i}=\Lambda$ and $D_{i}=D$ for all the subsystems. From Eqs. (5)-(7) we introduce $\lambda=\Lambda / \sqrt{D}$ and $\zeta\left(t_{j}\right)=s\left(t_{j}\right) / \sqrt{D}$. The input signal is subthreshold when $\zeta\left(t_{j}\right)<\lambda$ and suprathreshold when $\zeta\left(t_{j}\right)>\lambda$.

From Eqs. (4)-(7) the ensemble average of $Y_{N}\left(t_{j}\right)$ is calculated as

$$
\left\langle Y_{N}\left(t_{j}\right)\right\rangle=\frac{1}{2}\left[\operatorname{erfc}\left(\frac{\lambda-\zeta\left(t_{j}\right)}{\sqrt{2}}\right)-\operatorname{erfc}\left(\frac{\lambda+\zeta\left(t_{j}\right)}{\sqrt{2}}\right)\right] .
$$

The Taylor expansion of Eq. (9) around $\zeta\left(t_{j}\right)=0$ yields

$$
\left\langle Y_{N}\left(t_{j}\right)\right\rangle \approx G(\lambda) \zeta\left(t_{j}\right)+O\left(\zeta\left(t_{j}\right)^{3}\right),
$$

where $G(\lambda)$ represents the first-order coefficient, which is given by

$$
G(\lambda)=\left.\frac{\partial}{\partial \zeta}\left\langle Y_{N}\left(t_{j}\right)\right\rangle\right|_{\zeta\left(t_{j}\right)=0}=\sqrt{\frac{2}{\pi}} e^{-\lambda^{2} / 2} .
$$

Thus Eq. (10) shows that $\left\langle Y_{N}\left(t_{j}\right)\right\rangle$ is a linear function of the input signal $\zeta\left(t_{j}\right)$ for $\langle|\zeta|\rangle \ll 1$, where $\langle|\zeta|\rangle$ is the norm of the input signal, measured by the average of the amplitude of the signal. We call $G(\lambda)$ in Eq. (10) "gain."

Now, in order to measure the correlation between the input signal $s\left(t_{j}\right)$ and the output signal $Y_{N}\left(t_{j}\right)$ we introduce the normalized power norm [9],

$$
C_{1}=\frac{C_{0}}{\left[\overline{s\left(t_{j}\right)^{2}}\right]^{1 / 2}\left[\overline{\left(Y_{N}\left(t_{j}\right)-\overline{Y_{N}\left(t_{j}\right)}\right)^{2}}\right]^{1 / 2}},
$$

where $C_{0}$ is defined as

$$
C_{0}=\overline{s\left(t_{j}\right) Y_{N}\left(t_{j}\right)},
$$

with the overbar denoting an average over time, 


$$
\overline{s\left(t_{j}\right) Y_{N}\left(t_{j}\right)}=\lim _{M \rightarrow \infty} \frac{1}{M} \sum_{j=1}^{M} s\left(t_{j}\right) Y_{N}\left(t_{j}\right)
$$

Maximizing $C_{1}$ corresponds to maximizing the coherence between $s\left(t_{j}\right)$ and $Y_{N}\left(t_{j}\right)$, namely, it is equivalent to maximizing information transmission through the devices in Fig. 1.

At first, we discuss the numerator of Eq. (12). For large $N$, $Y_{N}\left(t_{j}\right)$ asymptotically tends to $\left\langle Y_{N}\left(t_{j}\right)\right\rangle$ according to the law of large number. From Eq. (10) the power norm $C_{0}$ is calculated as

$$
C_{0} \approx \frac{G(\lambda)}{\sqrt{D}}\|s\|^{2}
$$

where $\|s\|$ is defined as $\sqrt{\overline{s\left(t_{j}\right)^{2}}}$, namely, the power norm is proportional to the gain $G(\lambda)$ for a given input signal.

Next we consider the denominator of Eq. (12). For the purpose we introduce $\eta\left(t_{j}\right) \equiv\left\langle Y_{N}\left(t_{j}\right)\right\rangle-Y_{N}\left(t_{j}\right)$ we have $\left\langle\eta\left(t_{j}\right)\right\rangle=0$ and

$$
\begin{aligned}
\Delta_{j} & \equiv\left\langle\eta^{2}\left(t_{j}\right)\right\rangle=\left\langle\left[Y_{N}\left(t_{j}\right)-\left\langle Y_{N}\left(t_{j}\right)\right\rangle\right]^{2}\right\rangle=\left\langle Y_{N}^{2}\left(t_{j}\right)\right\rangle-\left\langle Y_{N}\left(t_{j}\right)\right\rangle^{2} \\
& =\frac{1}{N}\left\{P_{+}\left(t_{j}\right)+P_{-}\left(t_{j}\right)-\left[P_{+}\left(t_{j}\right)-P_{-}\left(t_{j}\right)\right]^{2}\right\} .
\end{aligned}
$$

$Y_{N}\left(t_{j}\right)$ is distributed around the ensemble average $\left\langle Y_{N}\left(t_{j}\right)\right\rangle$, and $\sqrt{\Delta_{j}}$ is of order of $N^{-1 / 2}$. Substituting Eqs. (5)-(7) into Eq. (16) yields

$$
\begin{aligned}
\Delta_{j}(N, \lambda)= & \frac{1}{N}\left\{\frac{1}{2}\left[\operatorname{erfc}\left(\frac{\lambda-\zeta\left(t_{j}\right)}{\sqrt{2}}\right)+\operatorname{erfc}\left(\frac{\lambda+\zeta\left(t_{j}\right)}{\sqrt{2}}\right)\right]\right. \\
& \left.-\frac{1}{4}\left[\operatorname{erfc}\left(\frac{\lambda-\zeta\left(t_{j}\right)}{\sqrt{2}}\right)-\operatorname{erfc}\left(\frac{\lambda+\zeta\left(t_{j}\right)}{\sqrt{2}}\right)\right]^{2}\right\} .
\end{aligned}
$$

Expanding the variance $\Delta_{j}$ around $\zeta\left(t_{j}\right)=0$ we have

$$
\Delta_{j}(N, \lambda)=\frac{1}{N}\left[\operatorname{erfc}\left(\frac{\lambda}{\sqrt{2}}\right)-\frac{1}{\pi} \exp \left(-\lambda^{2}\right) \zeta\left(t_{j}\right)^{2}\right]+O\left(\zeta\left(t_{j}\right)^{4}\right)
$$

The first term of Eq. (18) results from a fluctuation of the output signal $Y_{N}\left(t_{j}\right)$ without the input signal.

Now we consider $\left[Y_{N}\left(t_{j}\right)-\overline{Y_{N}\left(t_{j}\right)}\right]^{2}$, calculated as follows [17]. We have $\left[Y_{N}\left(t_{j}\right)-\overline{Y_{N}\left(t_{j}\right)}\right]^{2}=\overline{Y_{N}\left(t_{j}\right)^{2} Y_{N}\left(t_{j}\right)^{2}}$. Since we consider a zero-mean input signal $\overline{Y_{N}\left(t_{j}\right)}=0$. From $Y_{N}\left(t_{j}\right)$ $=\left\langle Y_{N}\left(t_{j}\right)\right\rangle+\eta\left(t_{j}\right)$ we have

$$
\overline{Y_{N}\left(t_{j}\right)^{2}}=\overline{\left\langle Y_{N}\left(t_{j}\right)\right\rangle^{2}}+2 \overline{\left\langle Y_{N}\left(t_{j}\right)\right\rangle \eta\left(t_{j}\right)}+\overline{\eta\left(t_{j}\right)^{2}}=\overline{\left\langle Y_{N}\left(t_{j}\right)\right\rangle^{2}}+\overline{\Delta_{j}},
$$

where we use $\overline{\left\langle Y_{N}\left(t_{j}\right)\right\rangle \eta\left(t_{j}\right)}=0$, which is proven by employing an ergodic assumption. Hence from Eqs. (13) and (19), $C_{1}$ is expressed by

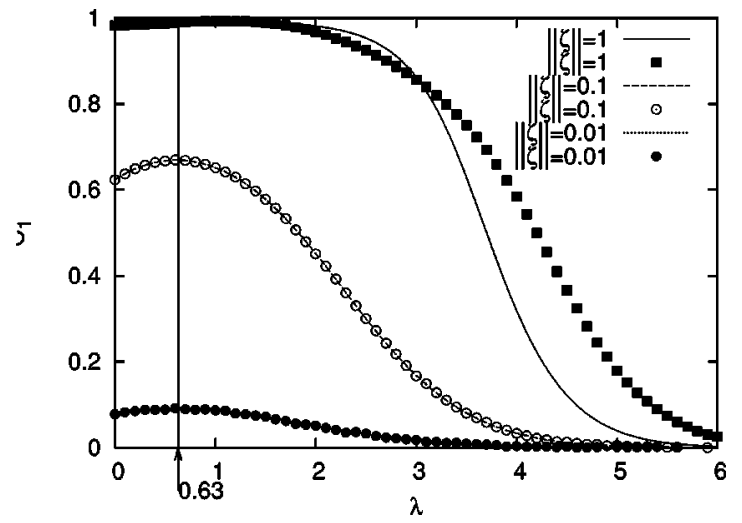

FIG. 2. The normalized power norm $C_{1}$, drawn as a function of the threshold value $\lambda$ at $N=100$ and a fixed amplitude of the input signal. We performed numerical simulations using the input signal given by $s\left(t_{j}\right)=0.5 A \sin \left(2 \pi f t_{j}\right)+A \cos \left(4 \pi f t_{j}\right)+0.25 A \sin \left(8 \pi f t_{j}\right)$ at $f=1.0$ and $T=0.001$. Then we have $\|s\| / D=\|\zeta\|=\sqrt{\frac{21}{32}} A / \sqrt{D}$. We calculate $C_{1}$ for various $\lambda$ at $\|\zeta\|=1,0.1$ and 0.01 . A solid curve represents the theoretical relation, Eq. (20) at $\|\zeta\|=1$, a dashed curve at $D=0.1$, a dotted curve at 0.1 , and a dashed curve at 0.01 . Filled squares are results of the numerical simulations at $\|\zeta\|=1$, unfilled circles at 0.1 , and filled circles at 0.01 . It is found that for $\|\zeta\|$ $=0.1$ and 0.01 , the value of $\lambda$ maximizing $C_{1}$ is 0.63 .

$$
C_{1}=\frac{G(\lambda)\|s\|^{2}}{\|s\| \sqrt{D\left[\overline{\left\langle Y_{N}\left(t_{j}\right)\right\rangle^{2}+\overline{\Delta_{j}}}\right]}}=\frac{1}{\sqrt{1+\frac{\overline{\Delta_{j}}}{\overline{\left\langle Y_{N}\left(t_{j}\right)\right\rangle^{2}}}}}=\frac{1}{\sqrt{1+c_{1}^{-1}}},
$$

where $c_{1}$ denotes the ratio between $\overline{\left\langle Y_{N}\left(t_{j}\right)\right\rangle^{2}}$ and the variance $\overline{\Delta_{j}}$,

$$
c_{1}=\frac{\overline{\left\langle Y_{N}\left(t_{j}\right)\right\rangle^{2}}}{\overline{\Delta_{j}}}
$$

This statistical measure, which is dimensionless and independent of scale, is the squared reciprocal of the coefficient of variation. High $c_{1}$ indicates low variability of the output signal. If $\left\langle Y_{N}\left(t_{j}\right)\right\rangle$ is approximated by the first term of Eq. (10), and $\bar{\Delta}_{j}$ the first term of Eq. (18), we obtain

$$
c_{1}=\frac{2 N}{\pi} \frac{\exp \left(-\lambda^{2}\right)}{\operatorname{erfc}\left(\frac{\lambda}{\sqrt{2}}\right)}\|\zeta\|^{2}
$$

where $\|\zeta\|=\|s\| / \sqrt{D}$. From Eq. (20) it is clear that $C_{1}$ is maximized when $c_{1}$ is maximized. Moreover, from Eq. (22) it is easily confirmed that $c_{1}$ is maximized at $\lambda \approx 0.63$, so that 0.63 is the optimal threshold.

In order to infer the input signal $s\left(t_{j}\right)$ from the output signal $Y_{N}\left(t_{j}\right)$ it is necessary that $\left\langle Y_{N}\left(t_{j}\right)\right\rangle$ is sufficiently larger than the fluctuation of $Y_{N}\left(t_{j}\right)$. If we impose the condition $c_{1}>1$, i.e., $C_{1}>1 / \sqrt{2}$ we have 

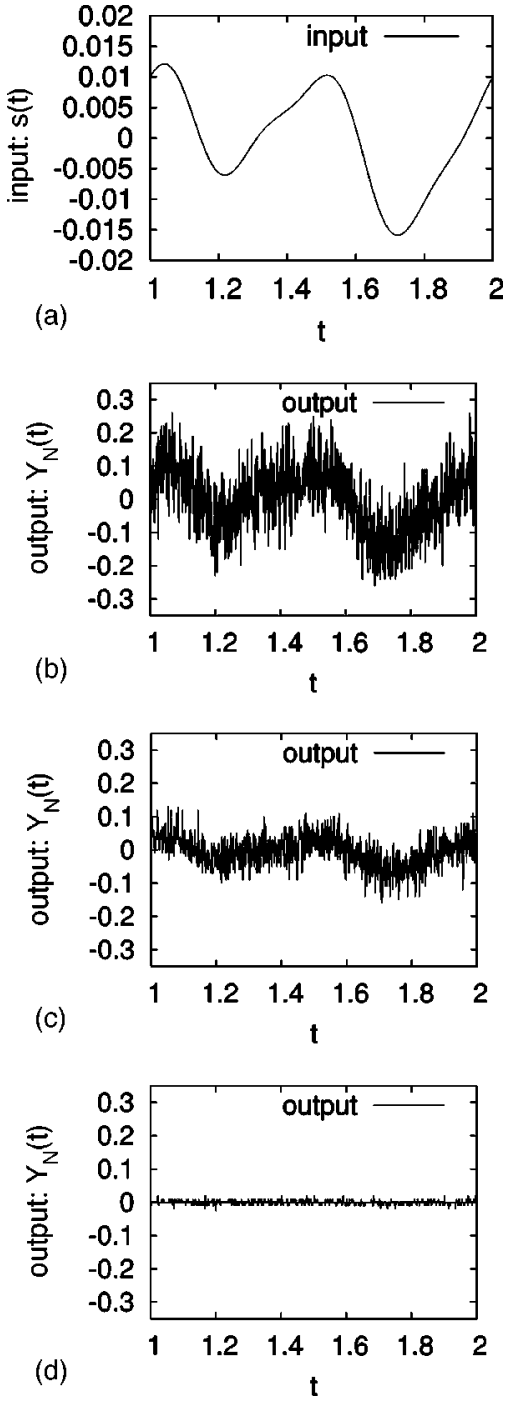

FIG. 3. Time series of the input signal $s\left(t_{j}\right)$, of which the wave form is the same as Fig. 2(a). We performed the numerical simulation at $N=100, D=0.1$, and $\|\zeta\|=0.1$. The output signal $Y_{N}\left(t_{j}\right)$ at (b) $\lambda=0.63\left(C_{1}=0.669\right)$, at (c) $\lambda=1.5\left(C_{1}=0.577\right)$, and (d) at $\lambda=3.0$ $\left(C_{1}=0.166\right)$.

$$
\|\zeta\|>\sqrt{\frac{\pi}{2 N}} \sqrt{\operatorname{erfc}\left(\frac{\lambda}{\sqrt{2}}\right)} \exp \left(\frac{\lambda^{2}}{2}\right) .
$$

This inequality assures that we can infer the input signal $s\left(t_{j}\right)$ from $\sqrt{D} Y_{N}\left(t_{j}\right) / G(\lambda)$ for $C_{1}>1 \sqrt{2}$.

\section{NUMERICAL SIMULATIONS}

Figure 2 displays the normalized power norm $C_{1}$ drawn as a function of $\lambda$ at fixed $\|\zeta\|$ from direct numerical simulations of the array of the bithreshold elements at $N=100$. The input signal is given by $s\left(t_{j}\right)=0.5 A \sin \left(2 \pi f t_{j}\right)+A \cos \left(4 \pi f t_{j}\right)$ $+0.25 A \sin \left(8 \pi f t_{j}\right)$ at $f=10.0$ and $T=0.001$, where $\|\zeta\|$ is given by $\sqrt{\frac{21}{32}} A / \sqrt{D}$. The points are obtained from the numerical simulations for various $\lambda$ at $\|\zeta\|=1,0.1$ and 0.01 , respectively. The curves represent Eq. (20) at the same parameters as the numerical simulations. It is found that the results from the numerical simulations are well fitted by the theoretical relation for $\|\zeta\|=0.1$ and 0.01 . $C_{1}$ has it maximum at $\lambda=0.63$.

However, for $\|\zeta\|=1$ it differs from the theoretical equation. The reason is because the output signal $Y_{N}\left(t_{j}\right)$ is not well approximated by the linear response of the input signal $s\left(t_{j}\right)$, due to the limit of applying the linear response theory. This disagreement is originated from difference between the nonlinear response of the system and the linear response assumed in Sec. III.

We demonstrate the output signal $Y_{N}\left(t_{j}\right)$ for various $\lambda$ $=0.63,1.5$, and 3.0 as shown in Fig. 3. The output signal $Y_{N}\left(t_{j}\right)$ is similar to the input signal $s\left(t_{j}\right)$ in the order for $\lambda$ shown. In this demonstration the input signal is given by a periodic signal. Naturally, the theoretical equation of $C_{1}$ that we obtained is applicable to any input signal (of course an aperiodic signal) satisfied with $\|\zeta\|<1$. Specifically, when the input signal is satisfied with Eq. (23) the output signal gives a good approximation of the input signal at $\lambda=0.63$.

\section{CONCLUSION}

We have investigated the parallel array of bithreshold elements both theoretically and numerically. We give an analytical approximation of the normalized power norm $C_{1}$ under the assumption that the norm of the input signal $s\left(t_{j}\right)$ is smaller than the standard deviation of the additive noises without distinguishing between subthreshold input and suprathreshold input. We confirmed that the theoretical approximation of $C_{1}$ is consistent with the results obtained from the direct numerical simulations of the array of the bithreshold elements when the norm of the input signal is smaller than the standard deviation of the additive noises. While for the larger norm than the standard deviation, the difference between the approximation and the numerical results appears. This disagreement is originated from the nonlinear response of the system. We demonstrated that the output signal gives a good approximation of the input signal at an appropriate threshold. We clarify that the optimal threshold, where the normalized power norm has a maximum value, is given by 0.63 times the standard deviation of the noises.

Our study may be applied to a sophisticated array of amplifiers. Moreover, the result shows that a collection of simple bithreshold sensors can detect a weak signal under an independently noisy environment. 
[1] R. Benzi, S. Sutera, and A. Vulpiani, J. Phys. A 14, L453 (1981).

[2] B. McNamara and K. Wiesenfeld, Phys. Rev. A 39, 4854 (1989).

[3] K. Wiesenfeld, D. Pierson, E. Pantazelou, C. Dames, and F. Moss, Phys. Rev. Lett. 72, 2125 (1994).

[4] C. Eichwald and J. Walleczek, Phys. Rev. E 55, R6315 (1997).

[5] L. Gammaitoni, P. Hänggi, P. Jung, and F. Marchesoni, Rev. Mod. Phys. 70, 223 (1998).

[6] N. G. Stocks, Phys. Rev. Lett. 84, 2310 (2000);N. G. Stocks, Phys. Rev. E 63, 041114 (2001);N. G. Stocks, Phys. Lett. A 279, 308 (2001).

[7] B. Xu, F. Duan, R. Bao, and J. Li, Chaos, Solitons Fractals 13, 633 (2002).

[8] L. Gammaitoni, F. Marchesoni, E. Menichella-Saetta, and S. Santucci, Phys. Rev. Lett. 62, 349 (1989);L. Gammaitoni, Phys. Rev. E 52, 4691 (1995).

[9] J. J. Collins, C. C. Chow, and T. T. Imhoff, Phys. Rev. E 52, R3321 (1995).

[10] F. Marchesoni, F. Apostolico, L. Gammaitoni, and S. Santucci,
Phys. Rev. E 58, 7079 (1998).

[11] A. C. H. Rowe and P. Etchegoin, Phys. Rev. E 64, 031106 (2001).

[12] F. Apostolico, L. Gammaitoni, F. Marchesoni, and S. Santucci, Phys. Rev. E 55, 36 (1997).

[13] M. Litong, Y. Hayakawa, and Y. Sawada, Phys. Rev. E 64, 026117 (2001).

[14] F. Marchesoni, L. Gammaitoni, and A. R. Bulsara, Phys. Rev. Lett. 76, 2609 (1996).

[15] J. F. Lindner, B. J. Breen, M. E. Wills, A. R. Bulsara, and W. L. Ditto, Phys. Rev. E 63, 051107 (2001).

[16] J. J. Collins, C. C. Chow, and T. T Imhoff, Nature (London) 376, 236 (1995).

[17] D. R. Chialvo, A. Longtin, and J. Müller-Gerking, Phys. Rev. E 55, 1798 (1997).

[18] N. G. Stocks and R. Mannella, Phys. Rev. E 64, 030902 (2001).

[19] M. I. Dykman, D. G. Luchinsky, R. Mannella, P. V.E. McClintock, H. E. Short, N. D. Stein, and N. G. Stocks, Phys. Lett. A 193, 61 (1994). 